\title{
MINIMIZATION OF THE GROUND STATE FOR TWO PHASE CONDUCTORS IN LOW CONTRAST REGIME*
}

\author{
CARLOS $\mathrm{CONCA}^{\dagger}$, ANTOINE LAURAIN $^{\ddagger}$, AND RAJESH MAHADEVAN ${ }^{\S}$
}

\begin{abstract}
In this article we consider the problem of the optimal distribution of two conducting materials with given volume inside a fixed domain, in order to minimize the first eigenvalue (the ground state) of a Dirichlet operator. It is known, when the domain is a ball, that the solution is radial, and it was conjectured that the optimal distribution of the materials consists of putting the material with the highest conductivity in a ball around the center. We show that this conjecture is not true in general. For this, we consider the particular case where the two conductivities are close to each other (low contrast regime) and we perform an asymptotic expansion with respect to the difference of conductivities. We find that the optimal solution is the union of a ball and an outer ring when the amount of the material with the higher density is large enough.
\end{abstract}

Key words. eigenvalue optimization, two-phase conductors, low contrast regime, asymptotic analysis

AMS subject classifications. Primary, 49Q10, 35P15; Secondary, 47J30, 49R05, 47A55

DOI. $10.1137 / 110847822$

1. Introduction. Optimal shape design for eigenvalues of elliptic operators provides a vast number of interesting and challenging mathematical problems; see [9] for an overview of the topic. In this paper we are considering the problem of minimizing the first eigenvalue of an elliptic operator with respect to the distribution of two conducting materials in a fixed domain.

Let $\Omega$ be a bounded domain in $\mathbb{R}^{d}$ which is to be called the design region. Let $m$ be a given positive number, $0<m<|\Omega|$, where $|\Omega|$ is the Lebesgue measure of the design region $\Omega$. Two materials with conductivities $\alpha$ and $\beta(0<\alpha<\beta)$ are distributed in arbitrary disjoint measurable subsets $A$ and $B$, respectively, of $\Omega$ so that $A \cup B=\Omega$ and $|B|=m$. Consider the two-phase eigenvalue problem

$$
\begin{aligned}
-\operatorname{div}(\sigma \nabla u) & =\lambda u \text { in } \Omega, \\
u & =0 \text { on } \partial \Omega,
\end{aligned}
$$

with the conductivity $\sigma=\alpha \chi_{A}+\beta \chi_{B}$. Let $\lambda$ be the first eigenvalue of (1.1)-(1.2)

\footnotetext{
* Received by the editors September 13, 2011; accepted for publication (in revised form) June 5, 2012; published electronically August 23, 2012.

http://www.siam.org/journals/siap/72-4/84782.html

†Departamento de Ingeniería Matemática (DIM) and Centro de Modelamiento Matemático (CMM), Universidad de Chile (UMI CNRS 2807), Casilla 170-3, Correo 3, Santiago, Chile and Institute for Cell Dynamics and Biotechnology: a Centre for Systems Biology, University of Chile, Santiago, Chile (cconca@dim.uchile.cl). This author's work was partially supported by the MICDB through grant ICM-P05-001-F, Fondap-Basal-Conicyt, and the Chilean and French governments through a CONICYT-INRIA grant.

¥Department of Mathematics, Humboldt University of Berlin, Berlin, Germany (laurain@math. hu-berlin.de). This author's work was supported by the Austrian Ministry of Science and Education and the Austrian Science Foundation FWF under START grant Y305 "Interfaces and Free Boundaries" and the subproject "Freelevel" of the SFB F32 "Mathematical Optimization and Applications in Biomedical Sciences."

$\S$ Facultad de Ciencias Físicas y Matemáticas, Universidad de Concepción, Concepción, Chile (rmahadevan@udec.cl). This author's work was supported by CONICYT-FONDECYT project 1090305.
} 
and let $u$ be the associated eigenvector. The variational formulation for $\lambda$ is

$$
\lambda=\min _{u \in H_{0}^{1}(\Omega)} \frac{\int_{\Omega} \sigma|\nabla u|^{2}}{\int_{\Omega} u^{2}}=\min _{u \in H_{0}^{1}(\Omega),\|u\|_{2}=1} \int_{\Omega} \sigma|\nabla u|^{2},
$$

where $\|u\|_{2}$ denotes the $L^{2}$-norm of $u$. In this paper the set $\Omega$ is fixed and we are interested in the dependence of $\lambda$ on $A$ and $B$. Since $A=\Omega \backslash B, \lambda$ may be described as a function of $B$ and we write $\lambda=\lambda(B)$. We consider the problem of minimizing $\lambda(B)$ with the constraint that the two phases are to be distributed in fixed proportions:

$$
\begin{aligned}
\operatorname{minimize} & \lambda(B) \\
\text { subject to } & B \in \mathcal{B},
\end{aligned}
$$

where

$$
\mathcal{B}=\{B \subset \Omega, B \text { measurable, }|B|=m\} .
$$

The existence of a solution to the problem (1.4)-(1.6) remains an open question. In general, one may find evidence of microstructural patterns in relation to minimizing sequences and the original problem may have to be relaxed to include microstructural designs. Existence of a solution and optimality conditions in the class of relaxed designs has been discussed in Cox and Lipton [4]. However, the original problem (1.4)-(1.6) may still have a solution for particular geometries as is the case when $\Omega$ is a ball. When $\Omega=\mathbb{B}(0, R)$ is a ball, the existence of a radially symmetric optimal set has been proved in [1], using rearrangement techniques and a comparison result for Hamilton-Jacobi equations and later, using only rearrangement techniques in [2]. Even in this case an explicit solution to the problem is not known. It was conjectured in $[2,3]$, for higher dimensions, that the solution $B^{*}$ to this problem is a ball $\mathbb{B}\left(0, R^{*}\right)$ like in the one-dimensional case [13], a result known since the 1950's. This conjecture has been recently reinforced by numerical tests in [3] and by the result in [5], where it is shown, using second order shape derivative calculus, that such a configuration is a local minimum for the problem when the volume constraint $m$ is small enough.

In spite of these results, we prove in this paper that the conjecture is not true in general. Indeed, the optimal domain $B^{*}$ cannot be a ball when $\alpha$ and $\beta$ are close to each other and $m$ is sufficiently large (cf. Theorem 2.9). The theoretical base for this result is provided by an asymptotic expansion of the eigenvalue with respect to $\beta-\alpha$ as $\beta \rightarrow \alpha$, which allows us to approximate (1.4)-(1.6) by a simpler optimization problem (cf. Corollary 2.4). This is done in section 2. Through this asymptotic formulation we are not only able to show that the previous conjecture is false but also we are able to compute numerical approximations of the solution in design domains other than balls. The numerical results are presented in section 4 . Another main feature of the paper is the proposal, in section 3, of a descent algorithm to solve the problem in general. This also permits establishing some necessary optimality conditions and allows us to deduce certain features of the optimal solution.

\section{Optimal sets for small conductivity gap.}

2.1. Asymptotic expansion. In this section we shall look at the problem of minimization of the first eigenvalue in the special case where the conductivities of the two materials, $\alpha$ and $\beta$, are close to each other (i.e., are in low contrast regime). 
Thus, we assume that $\beta=\beta^{\varepsilon}:=\alpha+\varepsilon$ with $\varepsilon>0$ converging eventually to zero. If the material with conductivity $\beta^{\varepsilon}$ occupies the subdomain $B$ of $\Omega$, the conductivity coefficient is, in this case,

$$
\sigma=\sigma^{\varepsilon}(B):=\alpha \chi_{A}+\beta^{\varepsilon} \chi_{B}=\alpha+\varepsilon \chi_{B} .
$$

Let $\lambda^{\varepsilon}(B)$ be the first eigenvalue in the problem

$$
\begin{aligned}
-\operatorname{div}\left(\sigma^{\varepsilon}(B) \nabla u^{\varepsilon}\right) & =\lambda^{\varepsilon}(B) u^{\varepsilon} \quad \text { in } \Omega, \\
u^{\varepsilon} & =0 \text { on } \partial \Omega
\end{aligned}
$$

for the conductivity $\sigma^{\varepsilon}(B)$. It is well known, from the Krel̆n-Rutman theorem [14], that the first eigenvalue of a linear elliptic operator is simple and the corresponding eigenfunction is of constant sign (and is the only eigenvalue whose eigenfunction does not change sign). So, we can choose the eigenfunction $u^{\varepsilon}=u^{\varepsilon}(B)$ corresponding to $\lambda^{\varepsilon}(B)$ to be positive and normalize it using the condition

$$
\int_{\Omega}\left(u^{\varepsilon}\right)^{2}=1
$$

In this way, $u^{\varepsilon}$ is uniquely defined. We affirm that, for fixed $B$, both $\lambda^{\varepsilon}(B)$ and $u^{\varepsilon}(B)$ depend analytically on the parameter $\varepsilon$. This result is classical in the perturbation theory of eigenvalues and follows readily, for instance, from Theorem 3, Chapter 2.5 of Rellich [17]. This justifies the ansätze

$$
\begin{aligned}
& \lambda^{\varepsilon}(B)=\lambda_{0}(B)+\varepsilon \lambda_{1}(B)+\cdots, \\
& u^{\varepsilon}(B)=v_{0}(B)+\varepsilon v_{1}(B)+\cdots .
\end{aligned}
$$

The convergence of the series in (2.6) holds in the space $H_{0}^{1}(\Omega)$. We first make some useful observations about the the terms in ansätze (2.5)-(2.6).

Proposition 2.1. In ansätze (2.5) and (2.6), the terms $\lambda_{0}(B)$ and $v_{0}(B)$ are independent of $B$. In fact, $\lambda_{0}(B)=\lambda_{0}$ is the first eigenvalue in the problem

$$
\begin{aligned}
-\alpha \Delta v_{0} & =\lambda_{0} v_{0} \quad \text { in } \Omega, \\
v_{0} & =0 \quad \text { on } \partial \Omega .
\end{aligned}
$$

The function $v_{0}$ is the positive eigenfunction corresponding to $\lambda_{0}$ and satisfies the normalization condition $\int_{\Omega} v_{0}^{2}=1$.

Proof. In view of the analytic dependence of $\lambda^{\varepsilon}(B)$ and $u^{\varepsilon}(B)$ on $\varepsilon$, it follows that $\lambda_{0}(B)$ is the limit, as $\varepsilon \rightarrow 0$, of $\lambda^{\varepsilon}(B)$ and that $v_{0}(B)$ is the limit of $u^{\varepsilon}(B)$ in $H_{0}^{1}(\Omega)$ as $\varepsilon \rightarrow 0$. Recalling that the eigenfunctions $u^{\varepsilon}(B)$ are positive it follows that $v_{0}(B)$ is nonnegative. Passing to the limit in (2.2)-(2.4), as $\varepsilon \rightarrow 0$, we obtain that $\lambda_{0}(B)$ and $v_{0}(B)$ solve the eigenvalue problem $(2.7)-(2.8)$ and $\int_{\Omega}\left(v_{0}(B)\right)^{2}=1$. As $v_{0}(B)$ is a positive eigenfunction, it follows by the Kreln-Rutman theorem that $\lambda_{0}$ is necessarily the first eigenvalue of the eigenvalue problem (2.7)-(2.8). Thus, $\lambda_{0}(B)$ and $v_{0}(B)$ are independent of $B$ and shall be denoted by $\lambda_{0}$ and $v_{0}$, respectively.

Proposition 2.2. In the ansatz $(2.5), \lambda_{1}(B)$ is given explicitly in terms of $v_{0}$ as follows:

$$
\lambda_{1}(B)=\int_{B}\left|\nabla v_{0}\right|^{2} d x
$$

Copyright $@$ by SIAM. Unauthorized reproduction of this article is prohibited. 
The following orthogonality relations hold true,

$$
\int_{\Omega} v_{0} v_{1}(B) d x=0=\int_{\Omega} \nabla v_{0} \cdot \nabla v_{1}(B) d x .
$$

Proof. The term $\lambda_{1}(B)$ in the ansatz (2.5) is the derivative of $\lambda_{\varepsilon}(B)$ with respect to $\varepsilon$ at $\varepsilon=0$, whereas the term $v_{1}(B)$ in the ansatz (2.6) is the derivative of $u^{\varepsilon}(B)$ with respect to $\varepsilon$ at $\varepsilon=0$. Differentiating (2.2)-(2.4) with respect to $\varepsilon$ at $\varepsilon=0$, we obtain the equations

$$
\begin{aligned}
-\operatorname{div}\left(\alpha \nabla v_{1}(B)\right)-\lambda_{0} v_{1}(B) & =\operatorname{div}\left(\chi_{B} \nabla v_{0}\right)+\lambda_{1}(B) v_{0} \text { in } \Omega \\
v_{1}(B) & =0 \text { on } \partial \Omega
\end{aligned}
$$

and the first of the orthogonality relations in (2.10). We have seen in Proposition 2.1 that $\lambda_{0}$ is the first eigenvalue of the problem (2.7)-(2.8) and is simple, the eigenspace being generated by the eigenfunction $v_{0}$. Taking $v_{1}(B)$ as a test function in (2.7)-(2.8) and using the first orthogonality relation in (2.10), we obtain the second orthogonality relation in (2.10). Finally, the system (2.11)-(2.12) admits a solution, by the Fredholm alternative, if and only if the right-hand side is orthogonal to the eigenfunction $v_{0}$. This condition leads to the relation

$$
\int_{\Omega} \operatorname{div}\left(\chi_{B} \nabla v_{0}\right) v_{0} d x+\lambda_{1}(B) \int_{\Omega} v_{0}^{2} d x=0 .
$$

As $\int_{\Omega} v_{0}^{2}=1$, we obtain

$$
\begin{aligned}
\lambda_{1}(B) & =-\int_{\Omega} \operatorname{div}\left(\chi_{B} \nabla v_{0}\right) v_{0} d x \\
& =-\int_{\partial \Omega} \chi_{B} v_{0} \nabla v_{0} \cdot n d S+\int_{B}\left|\nabla v_{0}\right|^{2} d x=\int_{B}\left|\nabla v_{0}\right|^{2} d x .
\end{aligned}
$$

Let us denote by

$$
\tilde{\lambda}^{\varepsilon}(B)=\lambda^{\varepsilon}(B)-\lambda_{0}-\varepsilon \lambda_{1}(B)
$$

the remainder in the ansatz (2.5). Although $\tilde{\lambda}^{\varepsilon}(B)$ is of order $\varepsilon^{2}$ for fixed $B$, we need estimates for $\tilde{\lambda}^{\varepsilon}(B)$ which are uniform with respect to $B$. This is given by the following theorem.

TheOREM 2.3. For $\varepsilon>0$ sufficiently small, there exists a constant $C$ independent of $\varepsilon$ and $B$ such that

$$
\left|\tilde{\lambda}^{\varepsilon}(B)\right| \leq C \varepsilon^{\frac{3}{2}} \quad \forall B \in \mathcal{B} .
$$

Proof. For the sake of clarity we divide the proof into several steps. Let $\varepsilon>0$ be a constant which is small compared to 1 . In what follows, $C_{1}, C_{2}, C_{3}, C_{4}$ will be constants independent of $\varepsilon$ and $B \in \mathcal{B}$.

Step 1 . We first show that

$$
\lambda^{\varepsilon}(B) \leq C_{1}, \quad\left\|u^{\varepsilon}(B)\right\|_{H_{0}^{1}(\Omega)} \leq C_{2} .
$$

Copyright $@$ by SIAM. Unauthorized reproduction of this article is prohibited. 
The first inequality in (2.14) follows readily from the variational characterization of $\lambda^{\varepsilon}(B)$. Indeed, we have

$$
\begin{aligned}
\lambda^{\varepsilon}(B) & =\inf \left\{\int_{\Omega} \sigma^{\varepsilon}(B) \nabla u \cdot \nabla u d x: u \in H_{0}^{1}(\Omega), \int_{\Omega} u^{2}=1\right\} \\
& \leq \frac{\alpha+1}{\alpha} \inf \left\{\int_{\Omega} \alpha \nabla u \cdot \nabla u d x: u \in H_{0}^{1}(\Omega), \int_{\Omega} u^{2}=1\right\} \\
& =\frac{\alpha+1}{\alpha} \lambda_{0}=C_{1},
\end{aligned}
$$

where we have used the variational characterization of $\lambda_{0}$. This proves the first estimate in (2.14). Now, using the uniform bound for $\lambda^{\varepsilon}(B)$ and using the fact that the coefficients $\sigma^{\varepsilon}(B)$ are uniformly bounded from below by $\alpha$, we have

$$
\alpha\left\|u^{\varepsilon}(B)\right\|_{H_{0}^{1}(\Omega)}^{2} \leq \int_{\Omega} \sigma^{\varepsilon}(B) \nabla u^{\varepsilon}(B) \cdot \nabla u^{\varepsilon}(B) d x=\lambda^{\varepsilon}(B) \leq C_{1} .
$$

Setting $C_{2}=C_{1} / \alpha$ we get the second estimate in (2.14).

Step 2. Next, we show that

$$
\left|\lambda^{\varepsilon}(B)-\lambda_{0}\right| \leq C_{3} \varepsilon \text {. }
$$

As $\sigma^{\varepsilon}(B) \geq \alpha$ for all $\varepsilon>0$, it follows from the variational characterization (2.15) of $\lambda^{\varepsilon}(B)$ that

$$
\lambda_{0} \leq \lambda^{\varepsilon}(B) \quad \forall \varepsilon>0 \text { and } \forall \text { measurable } B \subset \Omega .
$$

On the other hand, using the variational characterization (2.15), we have

$$
\begin{aligned}
\lambda^{\varepsilon}(B)-\lambda_{0} & \leq \int_{\Omega} \sigma^{\varepsilon}(B) \nabla v_{0} \cdot \nabla v_{0} d x-\int_{\Omega} \alpha \nabla v_{0} \cdot \nabla v_{0} d x \\
& =\varepsilon \int_{\Omega} \chi_{B} \nabla v_{0} \cdot \nabla v_{0} d x .
\end{aligned}
$$

So, after bounding further the right-hand side in (2.19), we have

$$
\lambda^{\varepsilon}(B)-\lambda_{0} \leq \int_{\Omega} \nabla v_{0} \cdot \nabla v_{0} d x \leq \frac{1}{\alpha} \lambda_{0} .
$$

The claim (2.17) follows from (2.18) and (2.20) by taking $C_{3}=\frac{1}{\alpha} \lambda_{0}$.

Step 3 . Now, we use the above estimate to show that

$$
\left\|u^{\varepsilon}(B)-v_{0}\right\|_{H_{0}^{1}(\Omega)} \leq C_{4} \sqrt{\varepsilon} .
$$

To begin with, we have

$$
\begin{aligned}
\lambda^{\varepsilon}(B)-\lambda_{0}= & \int_{\Omega} \sigma^{\varepsilon}(B) \nabla u^{\varepsilon}(B) \cdot \nabla u^{\varepsilon}(B) d x-\int_{\Omega} \alpha \nabla v_{0} \cdot \nabla v_{0} d x \\
= & \varepsilon \int_{\Omega} \chi_{B} \nabla u^{\varepsilon}(B) \cdot \nabla u^{\varepsilon}(B) d x \\
& +\alpha\left(\int_{\Omega} \nabla u^{\varepsilon}(B) \cdot \nabla u^{\varepsilon}(B) d x-\int_{\Omega} \nabla v_{0} \cdot \nabla v_{0} d x\right) \\
= & \varepsilon \int_{\Omega} \chi_{B} \nabla u^{\varepsilon}(B) \cdot \nabla u^{\varepsilon}(B) d x+2 \alpha \int_{\Omega} \nabla v_{0} \cdot \nabla\left(u^{\varepsilon}(B)-v_{0}\right) d x \\
& +\alpha \int_{\Omega} \nabla\left(u^{\varepsilon}(B)-v_{0}\right) \cdot \nabla\left(u^{\varepsilon}(B)-v_{0}\right) d x
\end{aligned}
$$

Copyright (c) by SIAM. Unauthorized reproduction of this article is prohibited. 
Rewriting the previous equality, we get

$$
\begin{aligned}
& \alpha \int_{\Omega}\left|\nabla\left(u^{\varepsilon}(B)-v_{0}\right)\right|^{2} d x+2 \alpha \int_{\Omega} \nabla v_{0} \cdot \nabla\left(u^{\varepsilon}(B)-v_{0}\right) d x \\
& =\lambda^{\varepsilon}(B)-\lambda_{0}-\varepsilon \int_{\Omega} \chi_{B} \nabla u^{\varepsilon}(B) \cdot \nabla u^{\varepsilon}(B) d x .
\end{aligned}
$$

Finally, we obtain

$$
\begin{aligned}
& \left.\left|\alpha \int_{\Omega}\right| \nabla\left(u^{\varepsilon}(B)-v_{0}\right)\right|^{2} d x+2 \alpha \int_{\Omega} \nabla v_{0} \cdot \nabla\left(u^{\varepsilon}(B)-v_{0}\right) d x \mid \\
& \leq\left|\lambda^{\varepsilon}(B)-\lambda_{0}\right|+\varepsilon\left|\int_{\Omega} \chi_{B} \nabla u^{\varepsilon}(B) \cdot \nabla u^{\varepsilon}(B) d x\right| \\
& \leq\left|\lambda^{\varepsilon}(B)-\lambda_{0}\right|+\varepsilon \frac{1}{\alpha} \lambda^{\varepsilon}(B) \\
& \leq \varepsilon\left(C_{3}+\frac{C_{1}}{\alpha}\right)
\end{aligned}
$$

where the last inequality is a consequence of (2.17) and (2.14). Dividing by $\varepsilon$ and passing to the limit in (2.23), we have

$$
\left|\lim _{\varepsilon \rightarrow 0} \alpha \frac{\int_{\Omega}\left|\nabla\left(u^{\varepsilon}(B)-v_{0}\right)\right|^{2} d x}{\varepsilon}+2 \alpha \int_{\Omega} \nabla v_{0} \cdot \nabla v_{1}(B) d x\right| \leq\left(C_{3}+\frac{C_{1}}{\alpha}\right) .
$$

In view of the second orthogonality relation in (2.10) we conclude that (2.21) holds with $C_{4}=C_{3}+\frac{C_{1}}{\alpha}$.

Step 4 . Finally, we show the estimate for the remainder $\tilde{\lambda}^{\varepsilon}(B)$

$$
\left|\tilde{\lambda}^{\varepsilon}(B)\right|=\left|\lambda^{\varepsilon}(B)-\lambda_{0}-\varepsilon \lambda_{1}(B)\right| \leq C \varepsilon^{\frac{3}{2}} .
$$

On the one hand, from the relation (2.19) and the definition of $\lambda_{1}(B)$ we observe that

$$
\lambda^{\varepsilon}(B)-\lambda_{0} \leq \varepsilon \lambda_{1}(B) .
$$

On the other hand, we have

$$
\begin{aligned}
\lambda_{0}+\varepsilon \lambda_{1}(B)-\lambda^{\varepsilon}(B) & \leq \int_{\Omega} \alpha \nabla u^{\varepsilon}(B) \cdot \nabla u^{\varepsilon}(B) d x+\varepsilon \int_{\Omega} \chi_{B} \nabla v_{0} \cdot \nabla v_{0} d x \\
-\int_{\Omega} \sigma^{\varepsilon} \nabla u^{\varepsilon}(B) \cdot \nabla u^{\varepsilon}(B) d x & \\
\leq & \varepsilon \int_{\Omega} \chi_{B} \nabla v_{0} \cdot \nabla v_{0} d x-\varepsilon \int_{\Omega} \chi_{B} \nabla u^{\varepsilon}(B) \cdot \nabla u^{\varepsilon}(B) d x \\
= & \varepsilon \int_{\Omega} \chi_{B} \nabla\left(v_{0}-u^{\varepsilon}(B)\right) \cdot \nabla\left(v_{0}-u^{\varepsilon}(B)\right) d x \\
& +2 \varepsilon \int_{\Omega} \chi_{B} \nabla u^{\varepsilon}(B) \cdot \nabla\left(v_{0}-u^{\varepsilon}(B)\right) d x
\end{aligned}
$$

So, by the result of Step 3, it follows from (2.26) and (2.28) that

$$
\begin{aligned}
\left|\lambda_{0}+\varepsilon \lambda_{1}(B)-\lambda^{\varepsilon}(B)\right| & \leq \varepsilon\left\|v_{0}-u^{\varepsilon}(B)\right\|_{H_{0}^{1}(\Omega)}^{2}+2 C_{2} \varepsilon\left\|v_{0}-u^{\varepsilon}(B)\right\|_{H_{0}^{1}(\Omega)} \\
& \leq C_{3}^{2} \varepsilon^{2}+2 C_{2} C_{3} \varepsilon^{\frac{3}{2}} \\
& \leq C \varepsilon^{\frac{3}{2}}
\end{aligned}
$$

Copyright $@$ by SIAM. Unauthorized reproduction of this article is prohibited. 
with $C=2 C_{2} C_{3}+1$ as $\varepsilon$ is small. This completes the proof of the estimate (2.13).

Corollary 2.4. Let $\lambda^{\varepsilon}(B)$ be the first eigenvalue in (2.2)-(2.3), let $\lambda_{0}$ be the first eigenvalue of problem (2.7)-(2.8), and let $\lambda_{1}(B)$ be as in Proposition 2.2. Then, we have

$$
\left|\inf _{B \in \mathcal{B}} \lambda^{\varepsilon}(B)-\lambda_{0}-\varepsilon \inf _{B \in \mathcal{B}} \lambda_{1}(B)\right| \leq C \varepsilon^{\frac{3}{2}},
$$

where $C$ is a constant independent of $\varepsilon$ and $B \in \mathcal{B}$

Proof. The proof is immediate from the estimate (2.13).

Corollary 2.5. If $B_{\varepsilon}^{\star} \in \mathcal{B}$ is a minimizer of $\lambda^{\varepsilon}(\cdot)$, then we have the following estimate:

$$
\left|\lambda_{1}\left(B_{\varepsilon}^{\star}\right)-\inf _{B \in \mathcal{B}} \lambda_{1}(B)\right| \leq 2 C \varepsilon^{\frac{1}{2}},
$$

where the constant $C$ is as in Theorem 2.3 , independent of $\varepsilon$ and $B \in \mathcal{B}$.

Proof. Let $B_{\varepsilon}^{\star} \in \mathcal{B}$ be a minimizer for $\lambda^{\varepsilon}(B)$. Then, we have

$$
\begin{aligned}
& \varepsilon\left|\lambda_{1}\left(B_{\varepsilon}^{\star}\right)-\inf _{B \in \mathcal{B}} \lambda_{1}(B)\right| \\
& =\left|\left(\lambda^{\varepsilon}\left(B_{\varepsilon}^{\star}\right)-\lambda_{0}-\varepsilon \lambda_{1}\left(B_{\varepsilon}^{\star}\right)\right)-\left(\lambda^{\varepsilon}\left(B_{\varepsilon}^{\star}\right)-\lambda_{0}-\varepsilon \inf _{B \in \mathcal{B}} \lambda_{1}(B)\right)\right| \\
& \leq\left|\lambda^{\varepsilon}\left(B_{\varepsilon}^{\star}\right)-\lambda_{0}-\varepsilon \lambda_{1}\left(B_{\varepsilon}^{\star}\right)\right|+\left|\inf _{B \in \mathcal{B}} \lambda^{\varepsilon}(B)-\lambda_{0}-\varepsilon \inf _{B \in \mathcal{B}} \lambda_{1}(B)\right| \\
& \leq 2 C \varepsilon^{\frac{3}{2}},
\end{aligned}
$$

where the inequalities follow from (2.25) and (2.29). Thus, we have obtained (2.30).

Remark 1 . By the previous corollary we see that a minimizer for $\lambda^{\varepsilon}(\cdot)$ is approximately a minimizer for $\lambda_{1}(\cdot)$ when $\varepsilon$ is small. Using a similar argument, we can show that if $B^{*}$ is a minimizer of $\lambda_{1}(\cdot)$, then, for small $\varepsilon>0$,

$$
\left|\lambda^{\varepsilon}\left(B^{*}\right)-\inf _{B \in \mathcal{B}} \lambda^{\varepsilon}(B)\right| \leq 2 C \varepsilon^{\frac{3}{2}} .
$$

Thus, minimizers for $\lambda_{1}(\cdot)$ are nearly optimal for $\lambda^{\varepsilon}(\cdot)$ in the above sense. In addition, we can get a numerical approximation of the minimum value of $\lambda^{\varepsilon}(\cdot)$ following the estimate in Corollary 2.4 after explicitly minimizing $\lambda_{1}(\cdot)$ whose solution is provided by Theorem 2.6 below.

We prove the following theorem which provides a characterization of the minimizer of $\lambda_{1}(\cdot)$, in terms of the level sets of the gradient of $v_{0}$.

THEOREM 2.6. There exists $c^{*} \geq 0$ such that whenever $B$ is a measurable subset of $\Omega$ satisfying

$$
\left\{x:\left|\nabla v_{0}(x)\right|<c^{*}\right\} \subset B \subset\left\{x:\left|\nabla v_{0}(x)\right| \leq c^{*}\right\}
$$

and $|B|=m$, then $B$ is an optimal solution for the problem of minimizing $\lambda_{1}(B)$ over $B \in \mathcal{B}$.

Proof. Let $f(c):=\left|\left\{x \in \Omega:\left|\nabla v_{0}(x)\right| \leq c\right\}\right|, f$ is clearly an increasing function with $0 \leq f(c) \leq|\Omega|$. Let $c^{*}:=\inf \{c: f(c) \geq m\}$. We have $f\left(c^{*}\right) \geq m$, indeed, let 
$c_{k}>c^{*}$ be a decreasing sequence such that $c_{k} \rightarrow c^{*}$. On one hand $f\left(c_{k}\right) \geq m$ and $\lim _{k \rightarrow \infty} f\left(c_{k}\right) \geq m$. On the other hand

$$
\begin{aligned}
\lim _{k \rightarrow \infty} f\left(c_{k}\right) & =\lim _{k \rightarrow \infty}\left|\left\{x \in \Omega:\left|\nabla v_{0}(x)\right| \leq c_{k}\right\}\right| \\
& =\left|\cap_{k \in \mathbb{N}}\left\{x \in \Omega:\left|\nabla v_{0}(x)\right| \leq c_{k}\right\}\right| \\
& =\left|\left\{x \in \Omega:\left|\nabla v_{0}(x)\right| \leq c^{*}\right\}\right|=f\left(c^{*}\right),
\end{aligned}
$$

so that $f\left(c^{*}\right) \geq m$. In a similar way we have $\left|\left\{x:\left|\nabla v_{0}(x)\right|<c^{*}\right\}\right| \leq m$. Indeed, let $c_{k}<c^{*}$ be an increasing sequence with $c_{k} \rightarrow c^{*}$. On one hand $f\left(c_{k}\right)<m$ and $\lim _{k \rightarrow \infty} f\left(c_{k}\right) \leq m$. On the other hand

$$
\begin{aligned}
\lim _{k \rightarrow \infty} f\left(c_{k}\right) & =\lim _{k \rightarrow \infty}\left|\left\{x \in \Omega:\left|\nabla v_{0}(x)\right| \leq c_{k}\right\}\right| \\
& =\left|\cup_{k \in \mathbb{N}}\left\{x \in \Omega:\left|\nabla v_{0}(x)\right| \leq c_{k}\right\}\right| \\
& =\left|\left\{x \in \Omega:\left|\nabla v_{0}(x)\right|<c^{*}\right\}\right|,
\end{aligned}
$$

so that $\left|\left\{x \in \Omega:\left|\nabla v_{0}(x)\right|<c^{*}\right\}\right| \leq m$.

If $B$ is a measurable set such that $\left\{x:\left|\nabla v_{0}(x)\right|<c^{*}\right\} \subset B \subset\left\{x:\left|\nabla v_{0}(x)\right| \leq c^{*}\right\}$ and $|B|=m$, then $B$ is an optimal solution for the problem of minimization of $\lambda_{1}(\cdot)$ over $\mathcal{B}$. Indeed if $D$ is any measurable subset of $\Omega$ such that $|D|=m$, we shall have $\left|B \cap D^{c}\right|=\left|B^{c} \cap D\right|$. Therefore,

$$
\begin{aligned}
\int_{D}\left|\nabla v_{0}\right|^{2} d x & =\int_{D \cap B}\left|\nabla v_{0}\right|^{2} d x+\int_{D \cap B^{c}}\left|\nabla v_{0}\right|^{2} d x \\
& \geq \int_{D \cap B}\left|\nabla v_{0}\right|^{2} d x+\int_{D^{c} \cap B}\left|\nabla v_{0}\right|^{2} d x=\int_{B}\left|\nabla v_{0}\right|^{2} d x
\end{aligned}
$$

as $\left|\nabla v_{0}\right| \geq c^{*}$ on $D \cap B^{c}$ whereas $\left|\nabla v_{0}\right| \leq c^{*}$ on $D^{c} \cap B$.

Remark 2. If $\left\{x:\left|\nabla v_{0}(x)\right|=c^{*}\right\}$ is of measure zero, then the unique solution (up to a set of measure zero) is the set $\left\{x:\left|\nabla v_{0}(x)\right|<c^{*}\right\}$ which shall also be open if $\Omega$ is a sufficiently smooth domain (as by Theorem 8.14 in [6] it can be concluded that $\nabla v_{0}$ is of class $C^{1}$ ). In view of Proposition 2.7, this will be the case when $\Omega$ is a disk.

2.2. Disproving the disk conjecture. When the domain $\Omega$ is a ball, in [3] it was conjectured, based on numerical tests, that the infimum of the first eigenvalue of (1.4)-(1.6) is attained when the material with the highest conductivity is placed in a concentric disk in the center of the domain. In this section, we prove that this conjecture is false, at least in two or three dimensions, for $\beta$ close to $\alpha$ and when the proportion of $\beta$ is large enough.

Let us take, in particular, $\Omega=\mathbb{B}(0,1) \subset \mathbb{R}^{d}$ to be the ball of center 0 and radius 1. In this case, the solution $v_{0}$ of $(2.7)-(2.8)$ is radial and smooth. By setting $w_{0}(|x|):=v_{0}(x),(2.7)-(2.8)$ becomes, using the Laplacian in polar $(r, \theta)$ or spherical $(r, \theta, \varphi)$ coordinates, for $d=2,3$

$$
\begin{array}{r}
r^{2} w_{0}^{\prime \prime}(r)+(d-1) r w_{0}^{\prime}(r)+r^{2} \frac{\lambda_{0}}{\alpha} w_{0}(r)=0, \\
w_{0}^{\prime}(0)=0, w_{0}(1)=0,
\end{array}
$$

where the boundary conditions (2.33) correspond to the continuity of the gradient at the origin and the Dirichlet condition on the boundary, respectively. The solution of 
this equation is

$$
\begin{array}{ll}
w_{0}(r)=J_{0}\left(\eta_{d} r\right) & \text { if } d=2, \\
w_{0}(r)=j_{0}\left(\eta_{d} r\right) & \text { if } d=3,
\end{array}
$$

where $J_{0}$ and $j_{0}$ denote Bessel functions of the first and second kind, respectively, and $\eta_{d}(d=2,3)$ are their respective zeros. The behavior of $w_{0}$ is depicted in Figure 1.
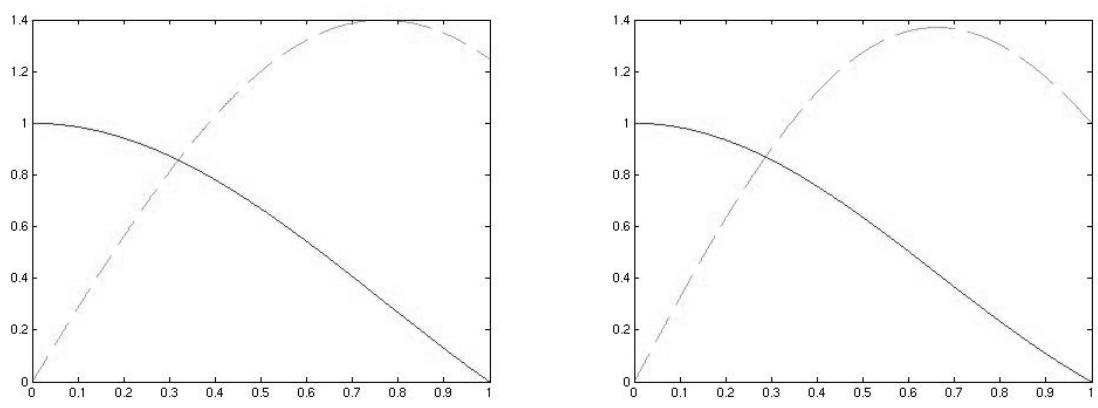

Fig. 1. Functions $w_{0}(r)$ (plain), and $w_{1}(r)=-w_{0}^{\prime}(r)$ (dashed) in dimensions $d=2$ (left) and $d=3$ (right). $r_{d}^{1}$ is such that $w_{1}$ is increasing on $\left[0, r_{d}^{1}\right]$ and decreasing on $\left[r_{d}^{1}, 1\right]$, and $r_{d}^{0}$ is such that $w_{1}\left(r_{d}^{0}\right)=w_{1}(1)$.

Proposition 2.7. For $\Omega=\mathbb{B}(0,1)$, the level set $\left\{\left|\nabla v_{0}\right|=c\right\}$ has zero measure for each $c \geq 0$.

Proof. Since $v_{0}(x)=w_{0}(|x|)$, we have $\nabla v_{0}(x)=w_{0}^{\prime}(|x|) x /|x|$ and consequently, we have

$$
\left|\nabla v_{0}\right|(x)=-w_{0}^{\prime}(|x|)
$$

Thus, the measure of the set $\left\{\left|\nabla v_{0}\right|(x)=c\right\}$ is positive if and only if the measure of one of the sets $\left\{x: w_{0}^{\prime}(|x|)=c\right\}$ or $\left\{x: w_{0}^{\prime}(|x|)=-c\right\}$ is positive. Thus, if $\left\{x: w_{0}^{\prime}(|x|)=\right.$ $-c\}$ has positive measure, this is the same as saying $S:=\left\{x: \nabla v_{0}(x)=-c \frac{x}{|x|}\right\}$ has positive measure. That is, the set $\left\{x: \nabla\left(v_{0}(x)+c|x|\right)=0\right\}$ has positive measure. By a classical result due to Morrey [16], if $\psi \in W_{\text {loc }}^{1,1}$, then $\nabla \psi=0$ almost everywhere on the set $\{\psi=0\}$. Applying this result to the function $\nabla\left(v_{0}+c|x|\right)$, we get

$$
\nabla^{2}\left(v_{0}+c|x|\right)=0
$$

almost everywhere on the set of positive measure $S$ defined above. So, now taking the trace we obtain

$$
-\Delta v_{0}(x)=\frac{c(d-1)}{|x|}
$$

on a set of positive measure. Then, by (2.7), this means that we have

$$
w_{0}(r)=\frac{\alpha c(d-1)}{\lambda_{0} r}
$$

on a set of positive measure which is clearly in violation of the behavior of the Bessel function $J_{0}$ or $j_{0}$. This concludes our proof. 
Let $\omega_{d}$ denote the volume of the unit ball, i.e., we have $\omega_{d}=\pi$ for $d=2$ and $\omega_{d}=4 \pi / 3$ for $d=3$ and let $r_{d}^{0}$ and $r_{d}^{1}$ be the constants mentioned in the caption of Figure 1 .

Proposition 2.8. When $\Omega$ is a ball, in two- or three-dimensional space, the unique symmetrical optimal domain $B^{*}$ which is solution of the minimization problem for $\lambda_{1}(B)$ over $B \in \mathcal{B}$ is of two possible types:

- Type I: If $m \leq \omega_{d}\left(r_{d}^{0}\right)^{d}$, then $B^{*}=\mathbb{B}\left(0,\left(m / \omega_{d}\right)^{1 / d}\right)$ or,

- Type II: If $m>\omega_{d}\left(r_{d}^{0}\right)^{d}$, then there exists $\xi^{0}$ and $\xi^{1}$ with

$$
\left(m / \omega_{d}\right)^{1 / d}<\xi^{0}<\xi^{1}<1
$$

and $B^{*}=\mathbb{B}\left(0, \xi^{0}\right) \cup\left(\mathbb{B}(0,1) \backslash \overline{\mathrm{B}\left(0, \xi^{1}\right)}\right)$.

Proof. In view of Theorem 2.6, Proposition 2.7, and Remark 2, a solution to the problem of minimization of $\lambda_{1}(\cdot)$ over $\mathcal{B}$ is the set $B^{*}=\left\{x \in \Omega:\left|\nabla v_{0}(x)\right| \leq c^{*}\right\}$, where $c^{*}$ is as in Theorem 2.6. Moreover, it is the unique solution (up to a set of measure zero). We may also write it as $B^{*}=\left\{x \in \Omega: w_{1}(|x|) \leq c^{*}\right\}$. Whenever $m \leq \omega_{d}\left(r_{d}^{0}\right)^{d}$, the equation $w_{1}(r)=c^{*}$ has exactly one solution $\xi$ in $(0,1)$. In this case the solution to our minimization problem is $B^{*}=\mathbb{B}(0, \xi)$ consisting of a single component in the center. The constraint $\left|B^{*}\right|=m$ implies $\xi=\left(m / \omega_{d}\right)^{1 / d}$.

In the case $m>\omega_{d}\left(r_{d}^{0}\right)^{d}$, the equation $w_{1}(r)=c^{*}$ has two solutions $\xi^{0}$ and $\xi^{1}$ in $(0,1)$. Then the solution set is

$$
B^{*}=\mathbb{B}\left(0, \xi^{0}\right) \cup\left(\mathbb{B}(0,1) \backslash \overline{\mathbb{B}\left(0, \xi^{1}\right)}\right)
$$

which completes our proof.

We now prove the main result of this section.

TheOrEm 2.9. When $\Omega=B(0,1)$, for $\beta=\alpha+\varepsilon$ sufficiently close to $\alpha$ and given an $m>\omega_{d}\left(r_{d}^{0}\right)^{d}$, the distribution of the materials wherein the material with the higher conductivity $\beta$ is placed in a concentric disk in the center of the domain is not optimal for the problem (1.4)-(1.5).

Proof. Our proof is based on Corollary 2.5 and Proposition 2.8. We shall assume that $m>\omega_{d}\left(r_{d}^{0}\right)^{d}$. Let $B^{\star}$ be the minimizer of $\lambda_{1}(\cdot)$ over $B \in \mathcal{B}$ which, by Proposition 2.8 , is the union of a ball $\mathbb{B}\left(0, \xi_{0}\right)$ and a ring or shell $\mathbb{B}(0,1) \backslash \overline{\mathrm{B}\left(0, \xi_{1}\right)}$. Let $B_{m}$ be the concentric ball in $\mathbb{B}(0,1)$ having the volume $m$. We show that $B_{m}$ cannot be the minimizer of $\lambda^{\varepsilon}(\cdot)$ in the problem (1.4)-(1.5) whenever $\beta=\alpha+\varepsilon$ is less than a certain quantity, to be precise, when

$$
2 C \varepsilon^{\frac{1}{2}}<\left|\lambda_{1}\left(B_{m}\right)-\lambda_{1}\left(B^{*}\right)\right| .
$$

For such an $\varepsilon>0$, if we assume that $B_{m}$ is a minimizer for $\lambda^{\varepsilon}(\cdot)$ in the problem (1.4)-(1.5), then, by (2.30) of Corollary 2.5, we obtain

$$
\left|\lambda_{1}\left(B_{m}\right)-\lambda_{1}\left(B^{*}\right)\right| \leq 2 C \varepsilon^{\frac{1}{2}} .
$$

This is clearly a contradiction. So, for $\varepsilon>0$ as above, $B_{m}$ cannot be a minimizer of $\lambda^{\varepsilon}(\cdot)$ in the problem (1.4)-(1.5).

Remark 3. Inequalities (2.36) and (2.14) actually gives the following upper bound:

$$
\varepsilon<\left(\frac{\left|\lambda_{1}\left(B_{m}\right)-\lambda_{1}\left(B^{*}\right)\right|}{2 C}\right)^{2}
$$

for the range of values of $\varepsilon$ for which Theorem 2.9 applies. A precise numerical approximation of this upper bound may be computed; see section 4.2. 
3. Descent algorithm and necessary optimality condition. In this section we consider domains $\Omega$ with any shape, and not only the case of a disk as in section 2.2. We also do not consider the low contrast regime assumption here, the algorithm that we describe in this section is valid for any values of $\alpha$ and $\beta$. We describe an algorithm which, starting from a given initial measurable set $B_{0}$, allows us to find a new measurable set $B_{1}$ such that the first eigenvalue $\lambda_{1}$ is decreased, i.e.,

$$
\lambda\left(B_{1}\right) \leq \lambda\left(B_{0}\right) .
$$

Algorithms based on a similar principle have been used successfully to minimize eigenvalues in problems with indefinite weight $[10,11]$. It may be used, as we shall see in subsection 4.2, to obtain numerical results. This algorithm allows us to derive necessary optimality conditions; see Corollary 3.3 , which in turn may be used to derive properties of the optimal set $B$ for particular geometries such as symmetric domains or polygonal domains. More details may be found at the end of this section.

Given an initial measurable set $B_{0}$, let $u_{B_{0}}$ and $\lambda\left(B_{0}\right)$ denote the first eigenvector and eigenvalue, respectively, for problem (1.1)-(1.2). Introduce the quantity

$$
\mathscr{M}\left(B_{0}, c\right)=\left|\left\{x:\left|\nabla u_{B_{0}}(x)\right| \leq c\right\}\right| .
$$

Lemma 3.1. The function $\mathscr{M}\left(B_{0}, c\right)$ is nondecreasing with respect to $c$ and is such that $\mathscr{M}\left(B_{0}, c\right) \rightarrow 0$ as $c \rightarrow 0$ and $\mathscr{M}\left(B_{0}, c\right) \rightarrow|\Omega|$ as $c \rightarrow \infty$. Furthermore, it is a right-continuous function. It is also left continuous at any $c$ if and only if the Lebesgue measure of $\left\{x:\left|\nabla u_{B_{0}}(x)\right|=c\right\}$ is zero.

Proof. The function $\mathscr{M}\left(B_{0}, c\right)$ is monotone nondecreasing due to the set inclusion $\left\{x:\left|\nabla u_{B_{0}}(x)\right| \leq c\right\} \subset\left\{x:\left|\nabla u_{B_{0}}(x)\right| \leq c^{\prime}\right\}$ whenever $c \leq c^{\prime}$. Due to the integrability of $\left|\nabla u_{B_{0}}\right|$, we have $\left|\left\{x:\left|\nabla u_{B_{0}}(x)\right|>c\right\}\right| \rightarrow 0$ as $c \rightarrow \infty$, and therefore, we can conclude that $\mathscr{M}\left(B_{0}, c\right) \rightarrow|\Omega|$ as $c \rightarrow \infty$. Also, $\mathscr{M}\left(B_{0}, c\right) \rightarrow 0$ as $c \rightarrow 0$ as it is known that the set of critical points for $u_{B_{0}}$ is of measure zero [15]. The right continuity may be seen from the following:

$$
\begin{aligned}
\mathscr{M}\left(B_{0}, c+\right)-\mathscr{M}\left(B_{0}, c\right) & =\lim _{c_{n} \searrow c} \mathscr{M}\left(B_{0}, c_{n}\right)-\mathscr{M}\left(B_{0}, c\right) \\
& =\lim _{c_{n} \searrow c}\left|\left\{x: c<\left|\nabla u_{B_{0}}(x)\right| \leq c_{n}\right\}\right| \\
& =\left|\cap_{n}\left\{x: c<\left|\nabla u_{B_{0}}(x)\right| \leq c_{n}\right\}\right| \\
& =0
\end{aligned}
$$

as the latter intersection is empty.

On the other hand,

$$
\begin{aligned}
\mathscr{M}\left(B_{0}, c\right)-\mathscr{M}\left(B_{0}, c-\right) & =\lim _{c_{n} \nearrow^{c}} \mathscr{M}\left(B_{0}, c\right)-\mathscr{M}\left(B_{0}, c_{n}\right) \\
& =\lim _{c_{n} \nearrow^{c}}\left|\left\{x: c_{n}<\left|\nabla u_{B_{0}}(x)\right| \leq c\right\}\right| \\
& =\left|\cap_{n}\left\{x: c_{n}<\left|\nabla u_{B_{0}}(x)\right| \leq c\right\}\right| \\
& =\left|\left\{x:\left|\nabla u_{B_{0}}(x)\right|=c\right\}\right|,
\end{aligned}
$$

which yields the assertion on left continuity.

Now define

$$
c_{0}:=\inf \left\{c: \mathscr{M}\left(B_{0}, c\right) \geq m\right\} .
$$

Copyright $@$ by SIAM. Unauthorized reproduction of this article is prohibited. 
As in the proof of Theorem 2.6, it may be shown that $\left|\left\{x:\left|\nabla u_{B_{0}}(x)\right| \leq c_{0}\right\}\right| \geq m$ and $\left|\left\{x:\left|\nabla u_{B_{0}}(x)\right|<c_{0}\right\}\right| \leq m$. Let $B_{1}$ be any measurable subset of $\Omega$ which satisfies $\left\{x:\left|\nabla u_{B_{0}}(x)\right|<c_{0}\right\} \subset B_{1} \subset\left\{x:\left|\nabla u_{B_{0}}(x)\right| \leq c_{0}\right\}$ and $\left|B_{1}\right|=m$.

We have the following result.

THEOREM 3.2. Given an initial measurable set $B_{0} \subset \Omega$ with $\left|B_{0}\right|=m$, let $c_{0}$ be as defined in (3.2) and let $B_{1}$ be a measurable set as above having measure $m$. Then we have $\lambda\left(B_{1}\right) \leq \lambda\left(B_{0}\right)$. Furthermore, if $\mathscr{M}\left(B_{0}, c\right)$ is continuous, then equality holds if and only if $B_{1}=B_{0}$ almost everywhere.

Proof. Consider the decompositions

$$
\begin{aligned}
& B_{0}=\left(B_{0} \cap B_{1}\right) \cup\left(B_{0} \cap B_{1}^{c}\right), \\
& B_{1}=\left(B_{0} \cap B_{1}\right) \cup\left(B_{0}^{c} \cap B_{1}\right) .
\end{aligned}
$$

Since $\left|B_{0}\right|=\left|B_{1}\right|=m$ we have with the above decompositions $\left|B_{0} \cap B_{1}^{c}\right|=\left|B_{0}^{c} \cap B_{1}\right|$. Noticing that $\left|\nabla u_{B_{0}}\right| \geq c_{0}$ on $B_{1}^{c}$ and $\left|\nabla u_{B_{0}}\right| \leq c_{0}$ on $B_{1}$, we may write

$$
\begin{aligned}
\int_{B_{0}}\left|\nabla u_{B_{0}}\right|^{2} & =\int_{B_{0} \cap B_{1}}\left|\nabla u_{B_{0}}\right|^{2}+\int_{B_{0} \cap B_{1}^{c}}\left|\nabla u_{B_{0}}\right|^{2} \\
& \geq \int_{B_{0} \cap B_{1}}\left|\nabla u_{B_{0}}\right|^{2}+c_{0}^{2}\left|B_{0} \cap B_{1}^{c}\right| \\
& =\int_{B_{0} \cap B_{1}}\left|\nabla u_{B_{0}}\right|^{2}+c_{0}^{2}\left|B_{0}^{c} \cap B_{1}\right| \\
& \geq \int_{B_{0} \cap B_{1}}\left|\nabla u_{B_{0}}\right|^{2}+\int_{B_{0}^{c} \cap B_{1}}\left|\nabla u_{B_{0}}\right|^{2}=\int_{B_{1}}\left|\nabla u_{B_{0}}\right|^{2} .
\end{aligned}
$$

Therefore

$$
\begin{aligned}
\lambda\left(B_{0}\right) & =\alpha \int_{\Omega}\left|\nabla u_{B_{0}}\right|^{2}+(\beta-\alpha) \int_{B_{0}}\left|\nabla u_{B_{0}}\right|^{2} \\
& \geq \alpha \int_{\Omega}\left|\nabla u_{B_{0}}\right|^{2}+(\beta-\alpha) \int_{B_{1}}\left|\nabla u_{B_{0}}\right|^{2} \\
& \geq \min _{u \in H_{0}^{1}(\Omega), \|\left. u\right|_{2}=1}\left(\alpha \int_{\Omega}|\nabla u|^{2}+(\beta-\alpha) \int_{B_{1}}|\nabla u|^{2}\right)=\lambda\left(B_{1}\right),
\end{aligned}
$$

and the inequality is proved.

Now, let us assume that $\mathscr{M}\left(B_{0}, c\right)$ is continuous. In view of Lemma 3.1, the continuity of $\mathscr{M}\left(B_{0}, c\right)$ implies that the set $\left\{x:\left|\nabla u_{B_{0}}(x)\right|=c_{0}\right\}$ has zero measure, so that $B_{1}$ can be taken to be $\left\{x:\left|\nabla u_{B_{0}}(x)\right| \leq c_{0}\right\}$. The equality $\lambda\left(B_{1}\right)=\lambda\left(B_{0}\right)$ holds if and only if the equality sign holds in (3.5), (3.6). In particular, the equality sign in (3.5) holds only if the equality sign holds in (3.3) and (3.4). Thus, as $\left|\nabla u_{B_{0}}(x)\right|>c_{0}$ on $B_{1}^{c}$, for the equality sign to hold in (3.3) we need that $\left|B_{0} \cap B_{1}^{c}\right|=0$. Consequently, we also have $\left|B_{0}^{c} \cap B_{1}\right|=0$. Thus, we have shown that $B_{1}=B_{0}$ except for a set of measure zero.

If $B_{1}=B_{0}$ except for a set of measure 0 , then clearly $\lambda\left(B_{1}\right)=\lambda\left(B_{0}\right)$.

The following corollary derives immediately from Theorem 3.2.

COROLlaRY 3.3 (necessary optimality condition). If a measurable set $B^{*}$ is optimal for the problem $(1.4)-(1.5)$ and if $\mathscr{M}\left(B^{*}, c\right)$ is continuous at $c^{*}$ for $c^{*}$ defined analogously as in (3.2) with $c^{*}$ replacing $c_{0}$ and $B^{*}$ replacing $B_{0}$, then up to a set of measure zero, $B^{*}$ is equal to the level set $\left\{x:\left|\nabla u_{B^{*}}(x)\right| \leq c^{*}\right\}$.

Copyright $\odot$ by SIAM. Unauthorized reproduction of this article is prohibited. 
Proof. It is enough to apply the previous theorem taking $B^{*}$ in place of $B_{0}, c^{*}$ instead of $c_{0}$, and then to take $B_{1}=\left\{x:\left|\nabla u_{B^{*}}(x)\right| \leq c^{*}\right\}$, which is allowed due to the continuity of $\mathscr{M}\left(B^{*}, \cdot\right)$ at $c^{*}$. Then, $\lambda\left(B_{1}\right) \leq \lambda\left(B^{*}\right)$ and $B^{*}$ is optimal, so we have the equality $\lambda\left(B_{1}\right)=\lambda\left(B^{*}\right)$. The conclusion follows, as by Theorem 3.2, equality holds only if $B_{1}$ is almost everywhere equal to $B^{*}$.

Assuming that the hypotheses of Corollary 3.3 are satisfied we obtain the following results for certain geometries.

The disk case. In the case $\Omega=\mathrm{B}(0, R)$ the optimal set $B^{*}$ should include the origin. Indeed, in [2], it is shown that the optimal domain is radially symmetric. The regularity and the radial symmetry of the solution imply that the gradient of $u$ vanishes at the origin 0 , and therefore, by Corollary 3.3, it follows that $0 \in B^{*}$.

The ring or torus case. It is expected that the solution is radically symmetric as in the disk case, then due to the Dirichlet condition and the positivity of the solution, it is clear that the gradient of $u$ vanishes at one point along a radius of the domain and by radisl symmetry, the gradient of $u$ vanishes on a whole circle whose center is the center of the ring or torus. Using Corollary 3.3 we obtain that this circle is in $B^{*}$. This property may be observed, for instance, in Figure 5 for different ratios $m /|\Omega|$.

Domains with corners in two dimensions. In this case the optimal set $B^{*}$ contains a neighborhood of the corners with angle smaller than $\pi$ while its complement $A^{*}=\Omega \backslash B^{*}$ contains a neighborhood of the corners with angle greater than $\pi$. Indeed, let $P \in \partial \Omega$ be a conical (corner) point of $\Omega$ and denote $\vartheta$ to be the associated angle at this corner. The classical theory of solution of elliptic partial differential equations in nonsmooth domains $[7,8,12]$ establishes that, in view of the Dirichlet boundary conditions, $u_{B^{*}}$ may be written as

$$
u_{B^{*}}(x)=c r^{\pi / \vartheta} \sin \left(\frac{\pi \theta}{\vartheta}\right)+u_{s}(x),
$$

where $c$ is the coefficient of the singularity which depends on the geometry of the domain, $\theta$ corresponds to polar coordinates with center $P$ and $\theta=0$ or $\theta=\vartheta$ on the tangents to $\partial \Omega$ at $P$, and $u_{s} \in H^{2}(\Omega)$ satisfies $u_{s}(x)=O\left(r^{\pi / \vartheta+1}\right)$ as $x \rightarrow P$. Therefore,

$$
\left|\nabla u_{B^{*}}(x)\right|=O\left(r^{\pi / \vartheta-1}\right) \text { as } x \rightarrow P
$$

and

$$
\left|\nabla u_{B^{*}}(x)\right| \rightarrow 0 \text { if } \vartheta<\pi, \quad\left|\nabla u_{B^{*}}(x)\right| \rightarrow \infty \text { if } \vartheta>\pi \quad \text { as } x \rightarrow P .
$$

Therefore, in view of Corollary 3.3, when $\vartheta<\pi$ we have $\left|\nabla u_{B^{*}}(x)\right| \rightarrow 0$ as $x \rightarrow P$, and we may find a small enough neighborhood of $P$ that will be included in $\{x$ : $\left.\left|\nabla u_{B^{*}}(x)\right| \leq c^{*}\right\}$ and thus also in $B^{*}$. When $\vartheta>\pi$ then $A^{*}=\Omega \backslash B^{*}$ contains a neighborhood of $P$ since $\left|\nabla u_{B^{*}}(x)\right| \rightarrow \infty$ as $x \rightarrow P$. These properties may be observed in Figures 2, 3, and 4.

Symmetrical domains. If the domain $\Omega$ has symmetries, then one can expect to show, as in [2], that the optimal domain has the same symmetries. Thus, if $\Omega \subset \mathbb{R}^{d}$ has $d$ independent hyperplanes of symmetry, it is expected that the solution set $B^{*}$ includes the point which is the intersection of these hyperplanes. If $\Omega$ is a square for instance, as in Figure 2, the center of this square belongs to $B^{*}$.

These properties are corroborated by numerical results shown in section 4.1 for such domains in the case when $\alpha$ and $\beta$ are close enough. 


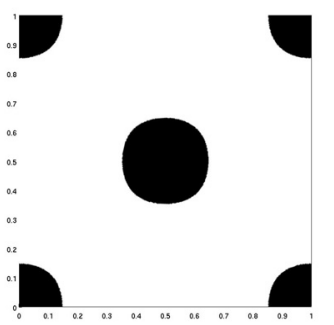

(a) $m /|\Omega|=0.1427$

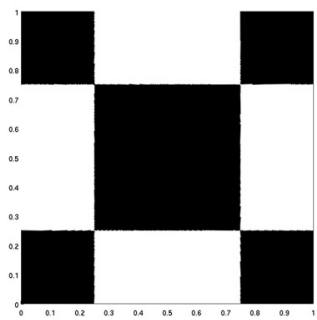

(c) $m /|\Omega|=0.5$

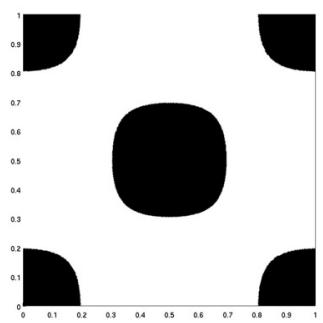

(b) $m /|\Omega|=0.2634$

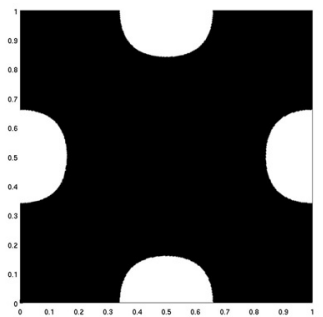

(d) $m /|\Omega|=0.8318$

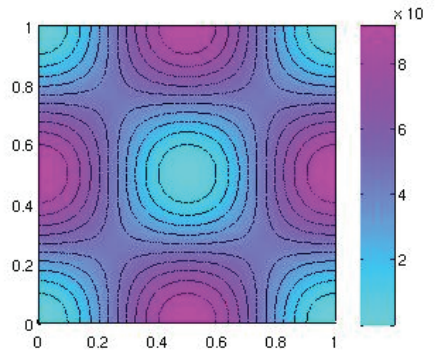

(e) isolines of $\left|\nabla v_{0}\right|^{2}$

Fig. 2. Nearly optimal distribution $B^{\star}$ in the square case. The dark region corresponds to $B$ and the material $\beta$, the white region to $A$ and material $\alpha$. As predicted by Corollary 3.3 , the optimal domain $B^{\star}$ contains the corners of the square, as well as the center since the gradient of the eigenfunction vanishes at that point. The domain is discretized using 319488 elements and 160385 nodes.

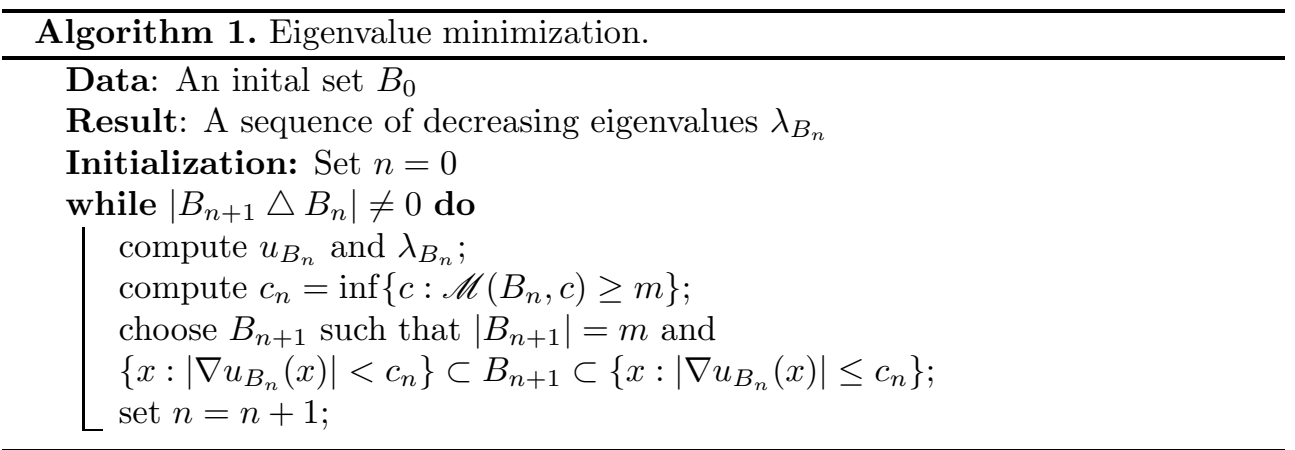




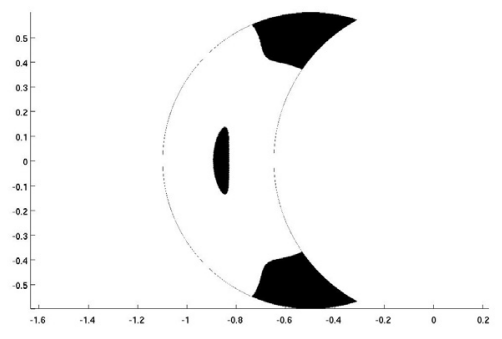

(a) $m /|\Omega|=0.2473$

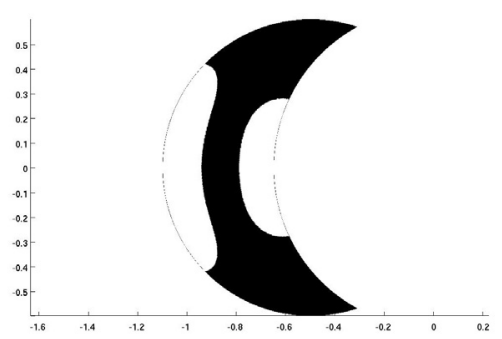

(c) $m /|\Omega|=0.6377$

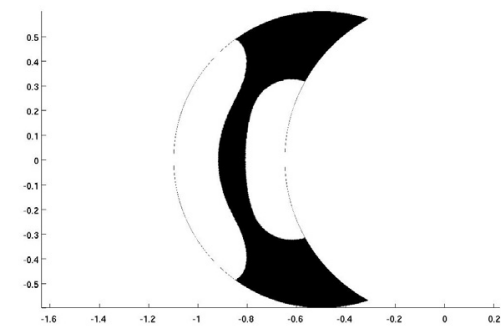

(b) $m /|\Omega|=0.4861$

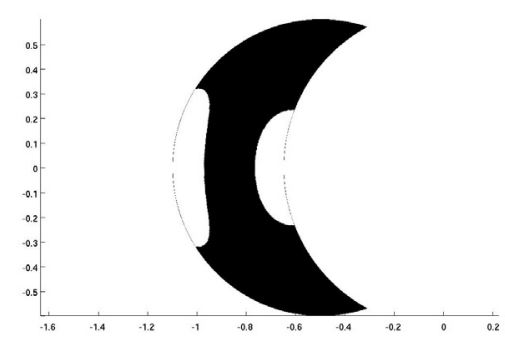

(d) $m /|\Omega|=0.7742$

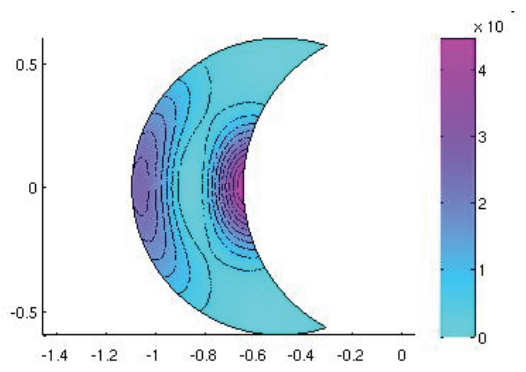

(e) isolines of $\left|\nabla v_{0}\right|^{2}$

FIG. 3. Nearly optimal distributions $B^{\star}$ in the crescent case for different values of $m$. The dark region corresponds to $B$ and the material $\beta$, the white region to $A$ and material $\alpha$. As predicted by Corollary 3.3, the approximately optimal domain $B^{\star}$ always contains the corners of the crescent and $a$ point in the center where the eigenfunction vanishes. The domain is discretized using 149504 elements and 75393 nodes.

To end this section, we propose the following algorithm based on Theorem 3.2 for the eigenvalue minimization problem. Its utility will be tested in section 4 . In the following, $B_{0} \triangle B_{1}:=\left(B_{0} \backslash B_{1}\right) \cup\left(B_{1} \backslash B_{0}\right)$ denotes the symmetric difference of two sets $B_{0}$ and $B_{1}$.

Remark 4. Algorithm 1 produces a sequence of domains $B_{n}$ such that

$$
\lambda\left(B_{n+1}\right) \leq \lambda\left(B_{n}\right) .
$$

However, it is not a priori guaranteed that $B_{n}$ converges to an admissible set $B^{*}$. 


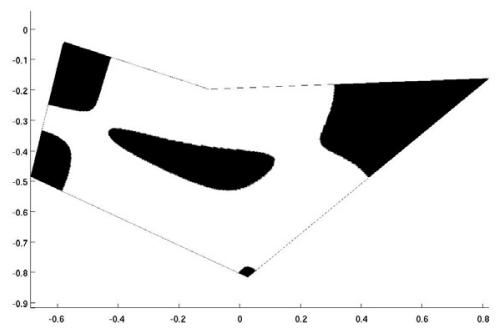

(a) $m /|\Omega|=0.3370$

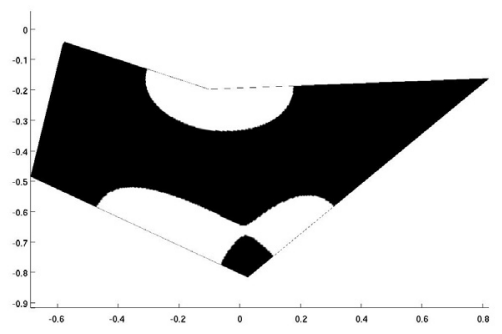

(c) $m /|\Omega|=0.7546$

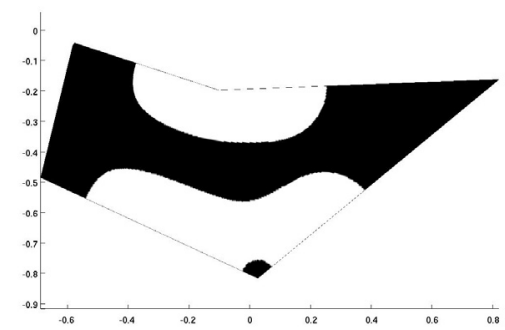

(b) $m /|\Omega|=0.5673$

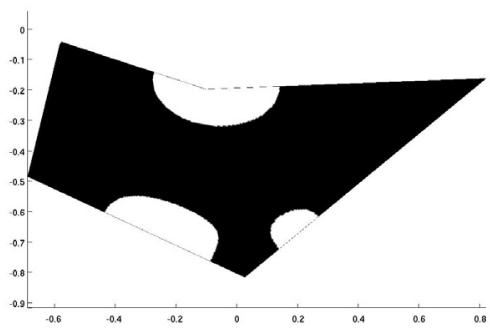

(d) $m /|\Omega|=0.8424$

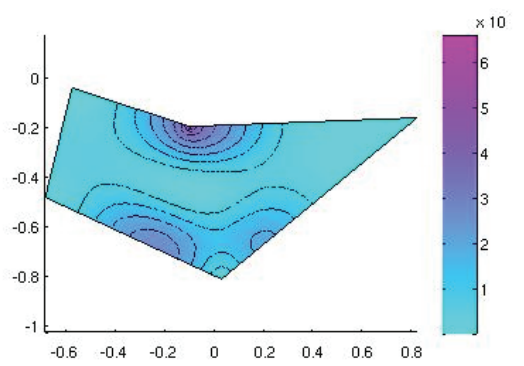

(e) isolines of $\left|\nabla v_{0}\right|^{2}$

FIG. 4. Nearly optimal distributions $B^{\star}$ in the polygon case for different values of $m$. The dark region corresponds to $B$ and the material $\beta$, the white region to $A$ and material $\alpha$. As predicted by Corollary 3.3 , the approximately optimal domain $B^{\star}$ contains the corners of the polygon with angle less than $\pi$ and a point in the center where the gradient of the eigenfunction vanishes. Note that $A^{*}=\Omega \backslash B^{*}$ contains a neighborhood of the point with the reentrant corner, i.e., the corner with angle greater than $\pi$. Indeed, in this corner the gradient is unbounded. The domain is discretized using 178176 elements and 89761 nodes.

Even if the algorithm converges to $B^{*}$, we cannot say that $B^{*}$ is a global or local optimum for the eigenvalue optimization problem. The computation of $c_{n}$ can be achieved by a binary search; see section 4 for a numerical realization of the algorithm in the case of spherical domain.

4. Numerical results. The aims of this section are twofold. On the one hand we would like to obtain approximate numerical solutions of problem (1.4)-(1.5) by 
applying Remark 1 and Theorem 2.6 in the case of nearly equal conductivities. On the other hand, in the general case, we would like to explore the numerical utility of the algorithm described in section 3 .

4.1. In low contrast regime. In this section we compute numerical approximations of solutions of the optimization problem (1.4)-(1.5) for general geometries $\Omega$, under the assumption of low contrast regime, i.e., $\beta=\beta^{\varepsilon}=\alpha+\varepsilon$ for small $\varepsilon$. Following Remark 1, the solution $B^{*}$ of the auxiliary problem corresponding to the minimization of $\lambda_{1}(\cdot)$ is then an approximate solution for (1.4)-(1.6) for small $\varepsilon$. In order to minimize $\lambda_{1}(\cdot)$ we use the result of Theorem 2.6, i.e., we look for a level $c^{*}$ and an optimal set $B^{*}$ satisfying $\left|B^{*}\right|=m$ and

$$
\left\{x:\left|\nabla v_{0}(x)\right|<c^{*}\right\} \subset B^{*} \subset\left\{x:\left|\nabla v_{0}(x)\right| \leq c^{*}\right\},
$$

where $v_{0}$ is the solution of (2.7)-(2.8). In our tests we observe that $\left\{x:\left|\nabla v_{0}(x)\right|=c^{*}\right\}$ is of measure zero and consequently we may apply Remark 2 to get

$$
B^{*}=\left\{x:\left|\nabla v_{0}(x)\right|<c^{*}\right\} .
$$

To compute $v_{0}$ we use a finite element discretization using piecewise linear elements. The domain is discretized by an unstructured triangular mesh using the PDE toolbox from MATLAB. The size of the grid for each test is indicated in the caption of the corresponding figure.

To obtain $B^{*}$, we compute the function $f(c)$ defined in the proof of Theorem 2.6 and look for $c^{*}$ such that $f\left(c^{*}\right)=m$. Numerically, this may be achieved by a binary search; see Algorithm 2 for a description of this algorithm. The set $B^{*}$ is then simply obtained by thresholding the function $\left|\nabla v_{0}\right|$ at $c^{*}$.

In Figures 2 to 6 , the minimizer $B^{*}$ for $\lambda_{1}(\cdot)$, which is nearly optimal for $\lambda^{\varepsilon}(\cdot)$ (for small $\varepsilon>0$ ), is plotted for various geometries $\Omega$ and values $m$. We also plot the isolines of $\left|\nabla v_{0}\right|^{2}$, where $v_{0}$ is the solution of (2.7)-(2.8), i.e., $v_{0}$ is the first eigenvector with constant conductivity $\alpha$. In all these examples, the features mentioned at the end of section 3 are seen to hold. For domains with salient angles, such as in Figures 2 to 4 , the set $B^{*}$ contains a neighborhood of these corners. On the contrary, reentrant corners such as in Figure 4 are always in $A^{*}$ since the gradient is unbounded at these points. We also observe that the set $B^{*}$ always contains a point (or more in the case of radial symmetry as in Figure 5) inside the domain. This interior point corresponds to the place where the gradient of $v_{0}$ vanishes and thus is expected to be in $B^{*}$ according to Corollary 3.3. For domains without corners, the optimal set may or may not touch the boundary as shown in Figures 5 and 6.

In general the optimal set $B^{*}$ seems to have a complex structure and a certain regularity, except for some particular values of $m$ as in Figure 2(c). We have checked that the nearly optimal distribution $B^{*}$ is stable with respect to the discretization of $v_{0}$ : the smoothness of $B^{*}$ obviously increases with the size of the mesh but the structure and shape of $B^{*}$ stay exactly the same. This stability is due to the smoothness of the eigenfunction $v_{0}$, the convergence of the finite element method and the fact that $\left|\left\{x:\left|\nabla v_{0}(x)\right| \leq c\right\}\right|$ is continuous in our tests.

4.2. The disk case for general $\boldsymbol{\beta}$. We apply Algorithm 1 of section 3 to obtain a numerical approximation of the optimal solution in the case of a disk for any value of $\beta$.

Since by the results of [1] the optimal solution exists in this domain and is radial, we may start with an initial distribution for $\beta$ which is radially symmetric. The 


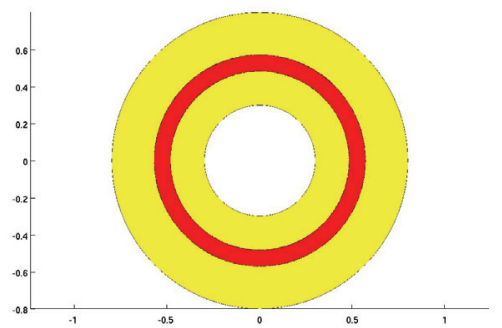

(a) $m /|\Omega|=0.1702$

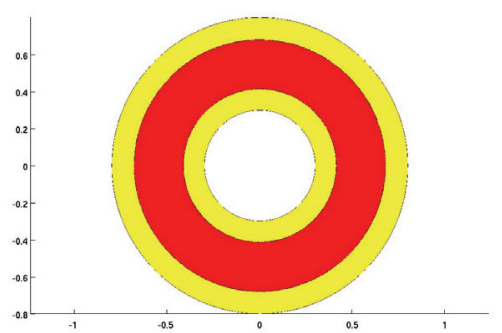

(c) $m /|\Omega|=0.5308$

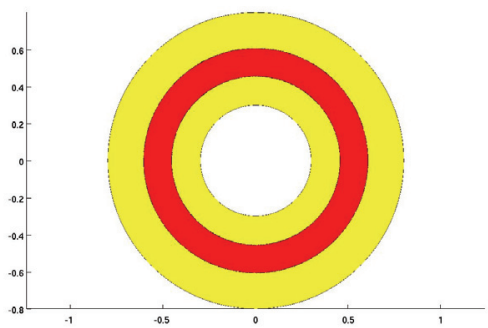

(b) $m /|\Omega|=0.2932$

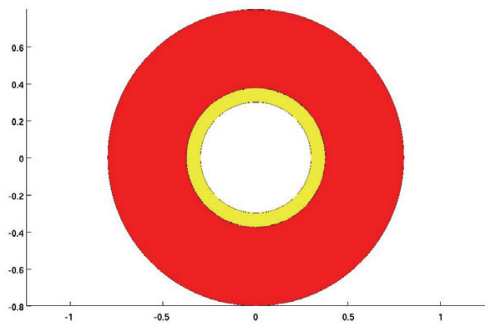

(d) $m /|\Omega|=0.9072$

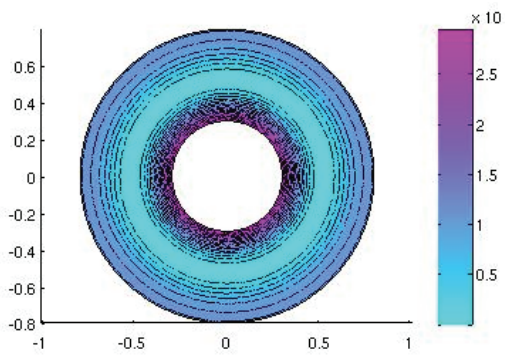

(e) isolines of $\left|\nabla v_{0}\right|^{2}$

Fig. 5. Nearly optimal distributions $B^{\star}$ in the ring case for different values of $m$. The dark region corresponds to $B$ and the material $\beta$, the bright region to $A$ and material $\alpha$. As predicted by Corollary 3.3, the approximately optimal domain $B^{\star}$ contains a ring at mid-distance to the two boundaries. It corresponds to the place where the gradient of the eigenfunction vanishes. For $m$ large enough, the domain $B^{*}$ touches the outer boundary of the ring, but it never touches the inner boundary, where the gradient attains its maximum (as long as $m<|\Omega|$ ). The domain is discretized using 966656 elements and 484928 nodes.

corresponding eigenfunction is radially symmetric. Thus, we use polar coordinates $(r, \theta)$ in $2 \mathrm{D}$ or spherical $(r, \theta, \phi)$ coordinates in $3 \mathrm{D}$ and $(1.1)$ reduces to the ordinary differential equation

$$
-\operatorname{div}(\sigma(r) \nabla u(r))=-r^{-(d-1)} \frac{d}{d r}\left[\sigma(r) r^{d-1} u^{\prime}(r)\right]=\lambda u(r) .
$$

This equation is discretized using a standard numerical scheme. We use a grid $\left\{u_{i}\right\}_{i=1}^{N}$ with $N$ points and stepsize $h=1 / N$. Intermediate value points $\sigma_{i+\frac{1}{2}}$ and $r_{i+\frac{1}{2}}$ have 


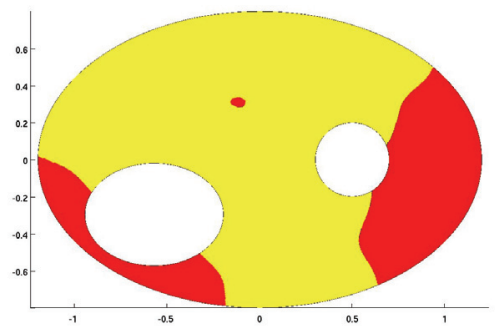

(a) $m /|\Omega|=0.2551$

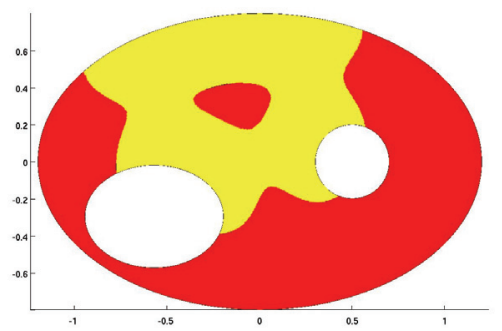

(c) $m /|\Omega|=0.5914$

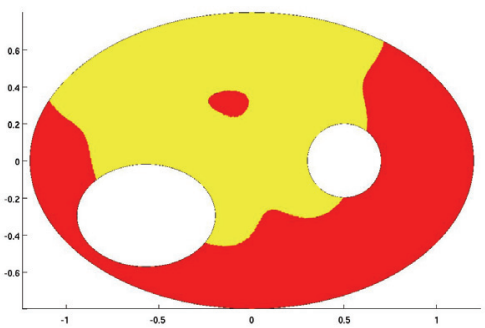

(b) $m /|\Omega|=0.4786$

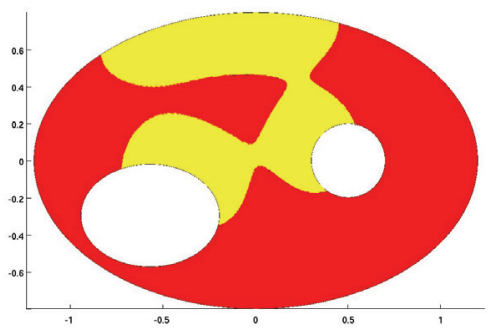

(d) $m /|\Omega|=0.7011$

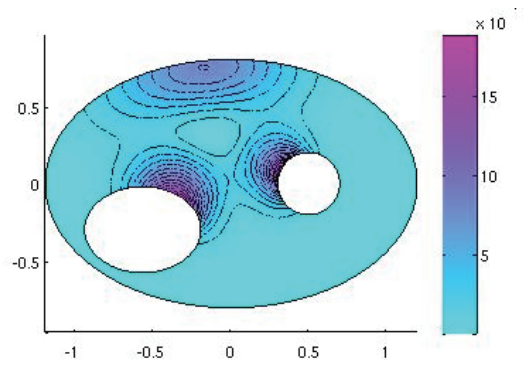

(e) isolines of $\left|\nabla v_{0}\right|^{2}$

FIG. 6. Nearly optimal distributions $B^{\star}$ in the case of an ellipse with two holes for different values of $m$. The dark region corresponds to $B$ and the material $\beta$, the bright region to $A$ and material $\alpha$. In this case we observe that the approximately optimal set $B^{*}$ has several connected components, except maybe for large $m$, and touches the boundary. The set $B^{*}$ always has a connected component in the center of the domain. This corresponds to a point where the gradient of the eigenfunction vanishes. The domain is discretized using 217088 elements and 109567 nodes.

to be determined. Using central differences we get the approximation

$$
\begin{aligned}
& \operatorname{div}(\sigma \nabla u)_{\mid r=r_{i}}=\left(r^{-(d-1)} \frac{d\left(\sigma(r) r^{d-1} u^{\prime}(r)\right)}{d r}\right)_{\mid r=r_{i}} \\
& \approx \frac{r_{i}^{-(d-1)}}{h^{2}}\left(u_{i+1}\left[\sigma_{i+\frac{1}{2}} r_{i+\frac{1}{2}}^{d-1}\right]-u_{i}\left[\sigma_{i+\frac{1}{2}} r_{i+\frac{1}{2}}^{d-1}+\sigma_{i-\frac{1}{2}} r_{i-\frac{1}{2}}^{d-1}\right]\right. \\
& \left.+u_{i-1}\left[\sigma_{i-\frac{1}{2}} r_{i-\frac{1}{2}}^{d-1}\right]\right) \text {. }
\end{aligned}
$$

Copyright $\odot$ by SIAM. Unauthorized reproduction of this article is prohibited. 
This approximation has to be modified according to boundary conditions for points close to the boundary of the domain in the discretization.

At any step of the algorithm, given $B_{n}$ radially symmetric, after numerically calculating the eigenfunction $u_{B_{n}}$, the threshold $c_{n}$, which allows us to determine the new set $B_{n+1}$ and defined as $c_{n}:=\inf \left\{c: \mathscr{M}\left(B_{n}, c\right) \geq m\right\}$, as in (3.2), is determined using the following binary search:

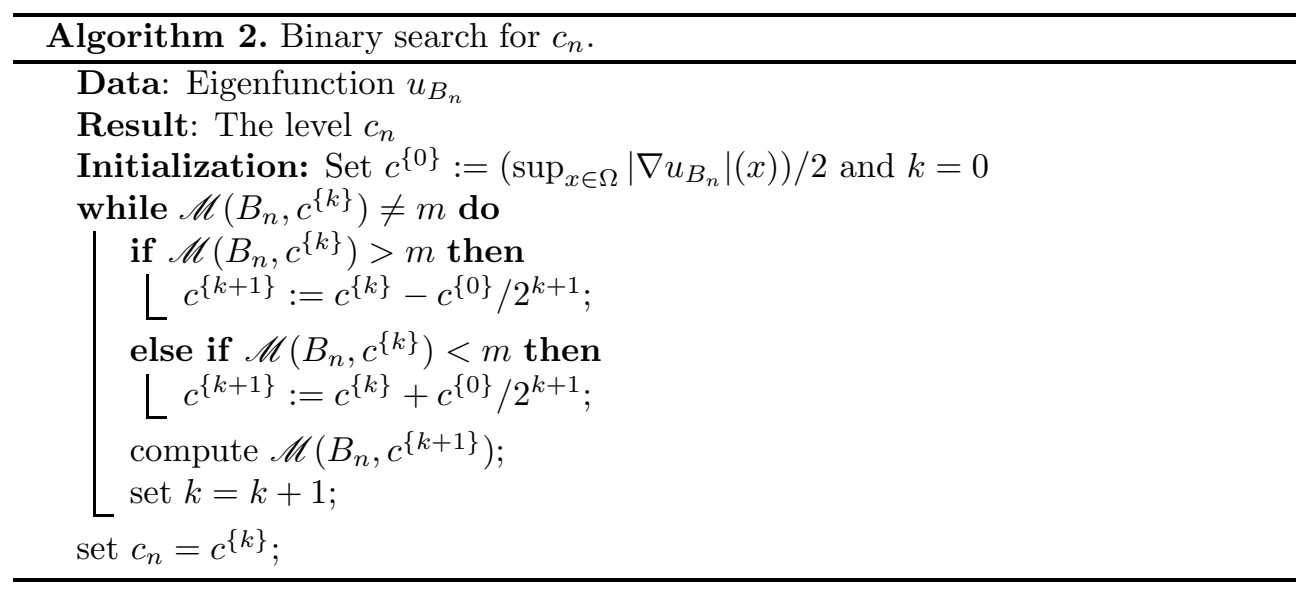

As shown in the proof of Theorem 3.2, since $\mathscr{M}\left(B_{n}, c\right)$ is observed to be continuous in our tests, we may then take $B_{n+1}=\left\{x:\left|\nabla u_{B_{n}}(x)\right|<c_{n}\right\}$. In this way, the radial symmetry of $B_{n}$ is also transmitted to $B_{n+1}$. In the discrete setting, the approximation of $\left|\nabla u_{B_{n}}\right|$ is only defined at grid points, and therefore, the new set $B_{n+1}$ resulting from Algorithm 2 satisfies the volume constraint $\left|B_{n+1}\right|=m$ only up to an error of order $h$. To find a more precise location of the set $B_{n+1}$, we linearly interpolate $\left|\nabla u_{B_{n}}\right|$ between the grid points. First, the set of grid points included in $B_{n+1}$ is determined using Algorithm 2. Then, if for instance the set $B_{n+1}$ is composed of one disk of radius $r_{1}$ and of one outer ring of radii $r_{2}$ and $r_{3}$ with $r_{2}<r_{3}<1$, writing down the volume constraint $\left|B_{n+1}\right|=m$ gives the equation $\pi\left(r_{1}^{2}+r_{3}^{2}-r_{2}^{2}\right)=m$ in $2 \mathrm{D}$. Using a linear interpolation of $\left|\nabla u_{B_{n}}\right|$ between the grid points, $r_{1}, r_{2}$, and $r_{3}$ become linear functions of $c_{n}$ and the volume constraint $\pi\left(r_{1}\left(c_{n}\right)^{2}+r_{3}\left(c_{n}\right)^{2}-r_{2}\left(c_{n}\right)^{2}\right)=m$ is a quadratic equation for the level $c_{n}$ which can be solved explicitly.

As commented in Remark 4, the convergence of the sequence $B_{n}$ is not guaranteed. However, in the following tests, for a given discretization level, we find that the condition $\left|B_{n+1} \triangle B_{n}\right|=0$ is always satisfied after a finite number of iterations and the algorithm converges. Even if the domains $B_{n}$ do converge to a domain $B^{*}$, this domain is not necessarily optimal for problem (1.4)-(1.5). Therefore, in order to find an optimal solution, we include a second step after Algorithm 1, wherein we make local perturbations to the $B^{*}$ in a descent direction using the shape derivative obtained in [3] (cf. the same for more details).

We present results in dimension $d=2$, and we take $\alpha=1, m=0.75^{2} \pi$, and $\Omega=\mathbb{B}(0,1)$. For the constant $r_{d}^{0}$ and $r_{d}^{1}$ appearing in Figure 1 and in Proposition 2.8 , we compute in dimension $d=2$ the numerical approximations $r_{2}^{0} \approx 0.53517$ and $r_{2}^{1} \approx 0.76562$. Note that $m \approx 1.7671>0.8998 \approx \omega_{d}\left(r_{d}^{0}\right)^{d}$ so that according to Proposition 2.8 and Theorem 2.9 we should find an optimum of type II when $\beta$ is small enough. The grid is made of $N=10^{4}$ points and the corresponding step size is 
$h=10^{-4}$. To explore the possibilities of the algorithm, we test with three different initial sets, each with a different topology:

$$
\begin{aligned}
& B_{1}=\mathbb{B}(0,0.75) \\
& B_{2}=\mathbb{B}(0,0.4) \cup\left(\mathbb{B}(0,0.8) \backslash \overline{\mathbb{B}\left(0, r_{2}\right)}\right) \text { with } r_{2}=\sqrt{0.4^{2}+0.8^{2}-0.75^{2}}, \\
& B_{3}=\mathbb{B}\left(0, r_{3}\right) \cup(\mathbb{B}(0,1) \backslash \overline{\mathbb{B}(0,0.8)}) \text { with } r_{3}=\sqrt{0.75^{2}-1+0.8^{2}} .
\end{aligned}
$$

For $\beta \leq 1.1$, starting with any of the three different initial guesses, the algorithm gives a solution $B_{\beta}$ of type II as predicted by Proposition 2.8. Precisely, $B_{\beta}$ is the union of a disk and a ring touching the outer boundary of $\Omega$ :

$$
B_{\beta}=\mathbb{B}\left(0, \xi_{\beta}^{0}\right) \cup \mathbb{B}(0,1) \backslash \overline{\mathbb{B}\left(0, \xi_{\beta}^{1}\right)} .
$$

In Table 4.1, $\xi_{\beta}^{0}$ and $\xi_{\beta}^{1}$ are given for values of $\beta$ close to $\alpha=1$, using $B_{1}$ as initial guess. Since $m>\omega_{d}\left(r_{d}^{0}\right)^{d}$ and according to Proposition $2.8, B^{*}=\mathbb{B}\left(0, \xi^{0}\right) \cup \mathbb{B}(0,1) \backslash \overline{\mathbb{B}\left(0, \xi^{1}\right)}$ is the global minimizer of $\lambda_{1}(B)$ and $\xi^{0}, \xi^{1}$ are the solutions of the equation $w_{1}(r)=c^{*}$. Numerically, we obtain the approximations $\xi^{0} \approx 0.62363$ and $\xi^{1} \approx 0.90907$. In Table 4.1 we observe that $\xi_{\beta}^{0} \rightarrow \xi^{0}$ and $\xi_{\beta}^{1} \rightarrow \xi^{1}$ as $\beta \rightarrow 0$.

TABLE 4.1

Optimal set $B_{\beta}$ and corresponding values of $\xi_{\beta}^{0}$ and $\xi_{\beta}^{1}$ for $1.01 \leq \beta \leq 1.1$ and eigenvalue $\lambda\left(B_{\beta}\right)$.

\begin{tabular}{cccccccc}
\hline$\beta$ & 1.0100 & 1.0250 & 1.0400 & 1.0550 & 1.0700 & 1.0850 & 1.1000 \\
\hline$\xi_{\beta}^{0}$ & 0.6231 & 0.6246 & 0.6258 & 0.6248 & 0.6255 & 0.6271 & 0.6265 \\
\hline$\xi_{\beta}^{1}$ & 0.9087 & 0.9097 & 0.9106 & 0.9099 & 0.9104 & 0.9115 & 0.9111 \\
\hline$\lambda\left(B_{\beta}\right)$ & 5.8105 & 5.8501 & 5.8890 & 5.9273 & 5.9649 & 6.0019 & 6.0383 \\
\hline
\end{tabular}

TABLE 4.2

Comparison of optimal sets and corresponding eigenvalues using the initial guesses $B_{2}$ or $B_{3}$ for $5.04 \leq \beta \leq 6.4$. The eigenvalue $\lambda_{\mathrm{I}}$ corresponds to the initial guess $B_{2}$ and $\lambda_{\mathrm{II}}$ corresponds to $B_{3}$. We observe that $\lambda_{\mathrm{I}}>\lambda_{\mathrm{II}}$ for these values of $\beta$.

\begin{tabular}{cccccccc}
\hline$\beta$ & 5.0400 & 5.2800 & 5.5200 & 5.7600 & 6.0000 & 6.2400 & 6.4000 \\
\hline$\xi_{\beta}^{0}$ & 0.7213 & 0.7252 & 0.7309 & 0.7347 & 0.7387 & 0.7426 & 0.7455 \\
\hline$\xi_{\beta}^{1}$ & 0.9786 & 0.9815 & 0.9857 & 0.9886 & 0.9915 & 0.9944 & 0.9966 \\
\hline$\lambda_{\mathrm{II}}$ & 8.9409 & 8.9885 & 9.0330 & 9.0740 & 9.1105 & 9.1450 & 9.1654 \\
\hline$\lambda_{\mathrm{I}}$ & 8.9470 & 8.9938 & 9.0367 & 9.0762 & 9.1127 & 9.1465 & 9.1677 \\
\hline
\end{tabular}

For $\beta>1.1$, the algorithm is more sensitive to the initial guess and we observe that the initial guesses $B_{1}$ and $B_{2}$ lead to a solution of type I (a disk of radius 0.75 in this example), while $B_{3}$ leads to a solution of type II (the union of a disk and of a ring touching the outer boundary). Denote by $\lambda_{I}:=\lambda(\mathbb{B}(0,0.75))$ the eigenvalue minimum obtained using the initial guesses $B_{1}$ or $B_{2}$, and by $\lambda_{I I}:=$ $\lambda\left(\mathbb{B}\left(0, \xi_{\beta}^{0}\right) \cup \mathbb{B}(0,1) \backslash \overline{\left.\mathbb{B}\left(0, \xi_{\beta}^{1}\right)\right)}\right.$ the eigenvalue minimum obtained using the initial guess $B_{3}$. In Table 4.2 we compare numerical computations of $\lambda_{\mathrm{I}}$ and $\lambda_{\mathrm{II}}$ for several values of $\beta$ and the corresponding values of $\xi_{\beta}^{0}$ and $\xi_{\beta}^{1}$. We observe that for $\beta \leq 6.4$ we have $\lambda_{\text {II }}<\lambda_{\text {I }}$ and the eigenvalues are getting very close to each other. For $\beta>6.4$ 
the three initial guesses give the same optimum of type I. We also observe that the domain $B_{\text {II }}$ itself numerically "converges" to $\mathrm{B}(0,0.75)$, in the sense that $\xi_{\beta}^{0} \rightarrow 0.75$ and $\xi_{\beta}^{1} \rightarrow 1$ as $\beta$ approaches the threshold value. These numerical results indicate that for $m=0.75^{2} \pi$ and $\alpha=1$, the approximate value $\beta \approx 6.4$ is a threshold above which the global optimal is always of type I (a disk) and below which the global optimum is either of type I or of type II, depending on the value of $m$, as in Proposition 2.8. This threshold probably depends on $m$ as well. The numerical approximation for the bound obtained in Remark 3 is $\varepsilon \leq 5.7 \times 10^{-9}$ for $m=0.75^{2} \pi$ but the numerical results indicate that the range of values of $\varepsilon$ for which Theorem 2.9 is valid is much larger.

Acknowledgments. The authors would like to thank the reviewers for their helpful comments. This research project was initiated during the workshop on partial differential equations and optimization at the Centro de Ciencias de Benasque Pedro Pascual, Spain. The authors thank the organizers for the fruitful conference.

\section{REFERENCES}

[1] A. Alvino, G. Trombetti, And P.-L. Lions, On optimization problems with prescribed rearrangements, Nonlinear Anal., 13 (1989), pp. 185-220.

[2] C. Conca, R. Mahadevan, and L. Sanz, An extremal eigenvalue problem for a two-phase conductor in a ball, Appl. Math. Optim., 60 (2009), pp. 173-184.

[3] C. Conca, R. Mahadevan, And L. Sanz, Shape derivative for a two-phase eigenvalue problem and optimal configurations in a ball, in CANUM 2008, ESAIM Proc. 27, EDP Sci., Les Ulis, France, 2009, pp. 311-321.

[4] S. Cox And R. Lipton, Extremal eigenvalue problems for two-phase conductors, Arch. Rational Mech. Anal., 136 (1996), pp. 101-117.

[5] M. Dambrine And D. Kateb, On the shape sensitivity of the first Dirichlet eigenvalue for two-phase problems, Appl. Math. Optim., 63 (2011), pp. 45-74.

[6] D. Gilbarg And N. S. Trudinger, Elliptic Partial Equations of Second Order, Classics Math., Springer-Verlag, Berlin, 2001.

[7] P. Grisvard, Elliptic Problems in Nonsmooth Domains, Monogr. Stud. Math. 24, Pitman (Advanced Publishing Program), Boston, MA, 1985.

[8] P. Grisvard, Singularities in Boundary Value Problems, Rech. Math. Appl. 22, Masson, Paris, 1992.

[9] A. Henrot, Extremum Problems for Eigenvalues of Elliptic Operators, Front. Math., Birkhäuser Verlag, Basel, 2006.

[10] M. Hintermüller, C.-Y. KaO, and A. Laurain, Principal Eigenvalue Minimization for an Elliptic Problem with Indefinite Weight and Robin Boundary Conditions, Technical Report 44, Karl-Franzens University of Graz, START-Project, Graz, Austria, 2011.

[11] C.-Y. KaO, Y. LOU, And E. Yanagida, Principal eigenvalue for an elliptic problem with indefinite weight on cylindrical domains, Math. Biosci. Eng., 5 (2008), pp. 315-335.

[12] V. A. Kondratiev, Boundary value problems for elliptic equations in domains with conical or angular points, Trudy Moskov. Mat. Obšč., 16 (1967), pp. 209-292.

[13] M. G. KREIN, On certain problems on the maximum and minimum of characteristic values and on the Lyapunov zones of stability. Amer. Math. Soc. Transl. (2), 1 (1955), pp. 163-187.

[14] M. G. Krein and M. A. Rutman, Linear operators leaving invariant a cone in a Banach space, Uspekhi Mat. Nauk, 3 (1948), pp. 3-95.

[15] H. LOU, On singular sets of local solutions to p-laplace equations, Chin. Ann. Math. Ser. B, 29 (2008), pp. 521-530.

[16] C. B. Morrey, Existence and differentiability theorems for the solutions of variational problems for multiple integrals, Bull. Amer. Math. Soc., 46 (1940), pp. 439-458.

[17] F. Rellich, Perturbation Theory of Eigenvalue Problems, Notes on Mathematics and its Applications, Gordon and Breach, New York, 1969. 